\title{
Selección de modelo de gestión tecnológica para pymes colombianas ${ }^{1}$ Selection of technology management model for colombian pymes
}

Seleção de modelo de gestão tecnológico para as pme colombianos

Recibido: octubre de 2012

Aprobado: diciembre de 2012
Rubén Darío Casas Duque ${ }^{2}$

Néstor Mauricio Urrego Urrego ${ }^{3}$

\section{Resumen}

Con base en el modelo Temaguide, los autores plantean la aplicación de un modelo de gestión tecnológica para mipymes colombianas. Este artículo condensa el estudio realizado en una empresa del sector manufacturero de la ciudad de Bogotá, en la cual se evidencia tanto la ausencia como la necesidad de administrar todo lo referente a la innovación. Se especifican también las herramientas que se pueden adaptar del modelo guía a la empresa, que son originadas en el planteamiento original del modelo Temaguide, pero acoplando solo las que por facilidad y poco complejidad brindan una solución efectiva a situaciones puntuales así como la alineación pertinente con la planeación estratégica de la empresa observada, permitiendo que se pueda realizar la relación entre el diagnóstico y el modelo. Por último, se plantean los indicadores que permitirán medir la efectividad del modelo, proyectando a su vez la aplicación en las mi pymes del país.

Palabras clave: gestión tecnológica, modelos de gestión tecnológica, innovación tecnológica, Temaguide, mi pymes.

\section{Abstract}

Based on the model Temaguide the authors present the application of a technology management model for mipymes Colombian. This article condenses the study of a manufacturing company in the city of Bogota, which is evident in both the absence and the need to manage everything related to innovation. Turn is specified tools model can be adapted to guide the company, these being caused in the original approach coupling model Temaguide but only those little complexity for ease and provide an effective solution to specific situations and the appropriate alignment with the strategic planning of the company observed, allowing it to make the relationship between diagnosis and model. Finally arises indicators to gauge the effectiveness model projecting turn mipymes application in the country.

Keywords: technology management, technology management models, innovation technology, Temaguide, mipymes.

1 Artículo de Investigación financiado por la Escuela Colombiana de Carreras Industriales (ECCI).

2 Escuela Colombiana de Carreras Industriales (ECCI), Grupo 9CN, Gestión Tecnológica 2013I, Ingeniería Industrial. Bogotá. - Colombia. Contacto: rubendarioc.d@gmail.com

3 Escuela Colombiana de Carreras Industriales (ECCI), Grupo 9CN, Gestión Tecnológica 2013I, Ingeniería Industrial. Bogotá - Colombia. Contacto: maourrego@gmail.com 


\section{Resumo}

Com base no modelo Temaguide os autores apresentam a aplicação de um modelo de gestão de tecnologia para MPMEs colombianos. Este artigo condensa o estudo de uma empresa de manufatura na cidade de Bogotá, que é evidente, tanto a ausência ea necessidade de gerenciar tudo relacionado à inovação. Por sua vez, é especificada ferramentas modelo pode ser adaptado para orientar a empresa, estes sendo causados na abordagem original de acoplamento modelo Temaguide mas apenas aqueles pouca complexidade para facilitar e proporcionar uma solução eficaz para situações específicas eo alinhamento apropriado com o planejamento estratégico da empresa observada, permitindo-lhe fazer a relação entre o diagnóstico eo modelo. Finalmente surge indicadores para avaliar o modelo de eficácia projetando aplicação mipymes vez no país.

Palavras-chave: gestão da tecnología, tecnologia de modelos de gestão, tecnología- inovação, Temaguide, MPMEs.

\section{Introducción}

La tendencia mundial de la globalización ha reformado los diferentes procesos en las empresas, no solo los modelos productivos, sino también las demás áreas de las organizaciones. El pensamiento sistémico de la organización ha hecho que estas áreas no se comporten de manera independiente, lo que vuelve indispensable que varios procesos se desarrollen de manera transversal; para que este funcionamiento ocurra es vital que la información fluya de forma adecuada.

El desarrollo de las tecnologías de información y comunicación (TIC) y su correspondiente aplicación en las industrias han permitido optimizar procesos, innovar en la prestación de servicios y modificar la gestión de las organizaciones en torno a la tecnología misma. La adquisición y el desarrollo de aplicaciones tecnológicas son solo una parte del proceso que permite a la empresa mejorar apoyada de herramientas en las que se implementen innovaciones tecnológicas. Por esto, la gestión tecnológica se hace necesaria dentro de la misma planeación estratégica de cualquier organización.

La gestión tecnológica se acopla a un proceso administrativo caracterizado por su capacidad de adaptarse a los cambios del entorno y aumentando su competitividad. Esta facilita actividades relacionadas con la valoración, desarrollo, adquisición, transferencia e implementación de los diversos avances que permitan dar una ventaja a cualquier tipo de organización.

Dada la importancia de mantenerse a la vanguardia para ser competitivos, contrastada por las falencias de las pymes en lo tecnológico y organizacional, se hace evidente la necesidad de estas de contemplar la aplicación de algún modelo de Gestión Tecnológica vinculado a su planeación estratégica, acorde a su situación actual, generadora de oportunidades de mejora y adaptable a los cambios externos. Se adaptó el modelo de Gestión Tecnológica propuesto por Temaguide, considerando que su funcionamiento y filosofía son paralelos a las necesidades contempladas para las pymes anteriormente.

\section{Metodología}

\section{Selección de la organización}

Para el estudio se seleccionó una organización de la cual se tuviera información de su situación actual y que cumpliera con unas características que permitieran replicar en un futuro las conclusiones obtenidas.

Características requeridas:

- Tamaño de empresa: mime según Ley 590 de 2000 y Ley 905 de 2004. 
- Tiempo de operación: 20-25 años.

- Ubicación: ciudad de alta concentración industrial en Colombia.

- Clasificación CIIU: sección C: Industrias manufactureras.

- Certificación ISO 9001/2008: no certificada.

\section{Selección del instrumento de medición}

Con el fin de determinar el estado actual de la organización, se aplicó un test de innovación empresarial diseñado por el Instituto Catalán de Tecnología. Este cuestionario fue aplicado a los líderes de cada proceso y su objeto es establecer los puntos fuertes y débiles de la organización referentes a la innovación.

\section{Selección del modelo de Gestión Tecnológica}

Después de analizar varios modelos de Gestión Tecnológica, se determinó seleccionar y adaptar el que satisficiera las siguientes características:

- Que reconozca la existencia de un ciclo iterativo de aprendizaje de la empresa.

- Que pueda aplicarse a proyectos específicos a la totalidad de la organización.

- De fácil integración con los procesos existentes en la empresa.

- Consolidado de aspectos valiosos de otros modelos.

- Que sea sistemático para anticipar los requisitos futuros y a la vez flexibles para responder a las necesidades urgentes.

\section{Resultados}

Con base en los resultados del test de innovación empresarial y el modelo de Gestión Tecnológica elegido y adaptado, se realizó un diagnóstico de la organización sobre su estado actual referente a

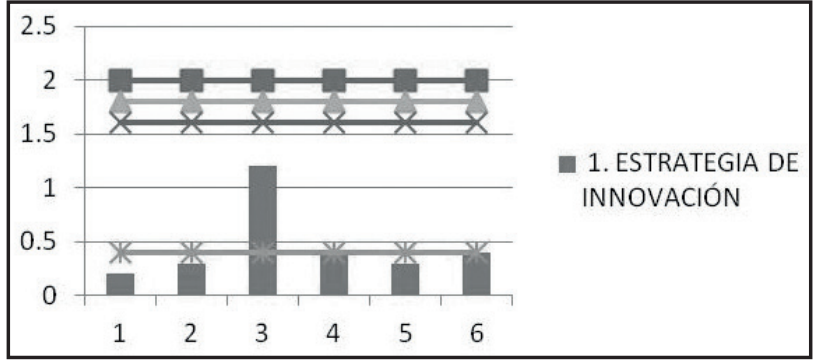

Gráfico 1. Estrategia de innovación

Fuente: elaboración propia.

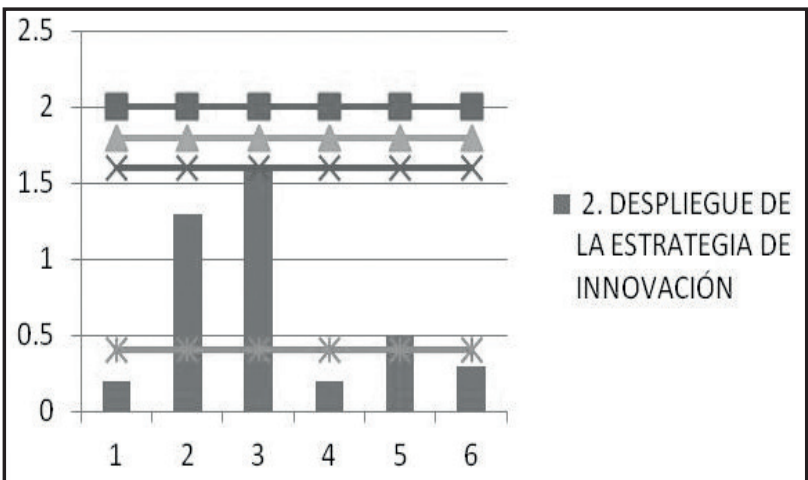

Gráfico 2. Despliegue de la estrategia de innovación.

Fuente: elaboración propia.

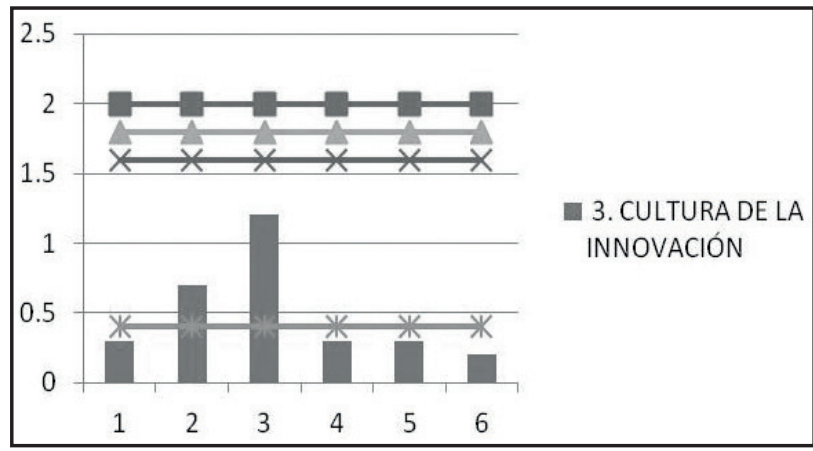

Gráfico 3. Cultura de la innovación

Fuente: elaboración propia.

la innovación. Se establecieron las posibles acciones para buscar la mejora en las actividades de la empresa. 


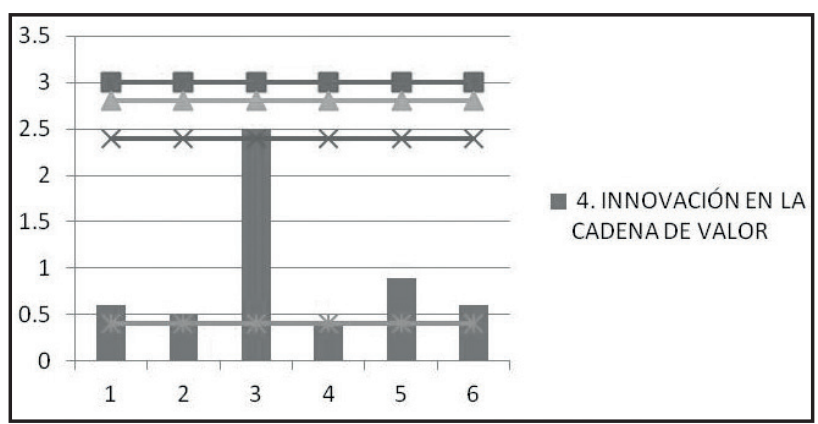

Gráfico 4. Innovación en la cadena de valor.

Fuente: elaboración propia.

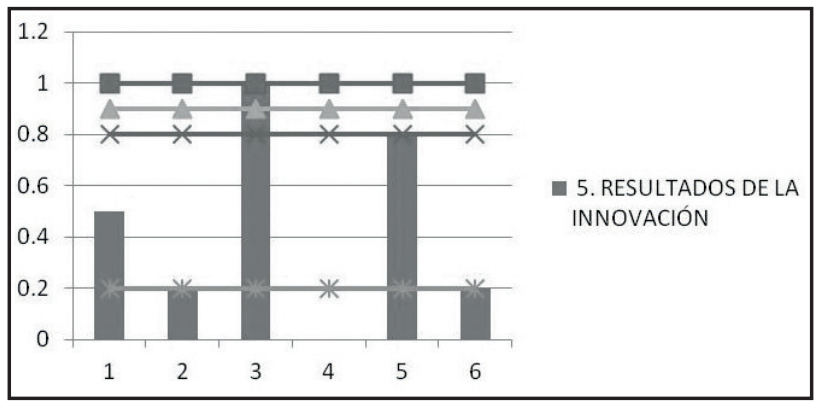

Gráfico 5. Resultados de la innovación.

Fuente: elaboración propia.

\section{Diagnóstico}

De la aplicación de las encuestas a cada líder de proceso se obtuvieron, después de su respectiva tabulación, unos resultados que llevaron a su análisis correspondiente. Se confirmó la carencia de una estructura o modelo de Gestión Tecnológica, la cual facilite el apropiamiento o desarrollo de nuevas tecnologías.

Se tuvieron en cuenta los apartes evaluados por el test de innovación empresarial, los cuales son: estrategia de la innovación, despliegue de la estrategia de innovación, cultura de la innovación, innovación de la cadena de valor y resultados de la innovación. Según las gráficas 1 a 5 , se puede notar que en la mayoría de estas solo se encuentra una de las encuestas (encuesta 3) con una alta calificación. Lo que contrasta con la lectura general de los jefes de área.
La encuesta número 3 corresponde al líder de proceso de producción, quien considera que a nivel general la empresa tiene un buen nivel de innovación; sin embargo, los jefes de calidad, operaciones, abastecimiento y comercial consideran que realmente la empresa dista mucho de una cultura innovadora. También se evidenció que a nivel general la compañía nunca ha generado un proceso para el desarrollo de nuevas ideas, incluso no lo ha contemplado dentro de su planeación estratégica y sus metas durante el tiempo que ha funcionado.

Lo expresado anteriormente causa falta de proyección de la compañía y sus productos como partícipes de un mercado global, derivado del poco interés de las directivas sobre el tema, lo cual se evidencia en la baja inversión en investigación y el poco interés en incentivar nuevas ideas tanto internas como externas.

Estos resultados justifican la necesidad de implementar en la empresa un modelo de gestión tecnológica, aplicable y funcional. Este debe permitir, además de adquirir las tecnologías adecuadas, el gestionar de manera apropiada en la organización dichas tecnologías.

\section{Modelo de gestión tecnológica para las U}

Después de analizar modelos de gestión tecnológica de diferentes autores, se estableció elegir la propuesta de la Fundación Cotec para la Innovación Tecnológica como modelo de gestión tecnológica para la empresa en la que se basa el

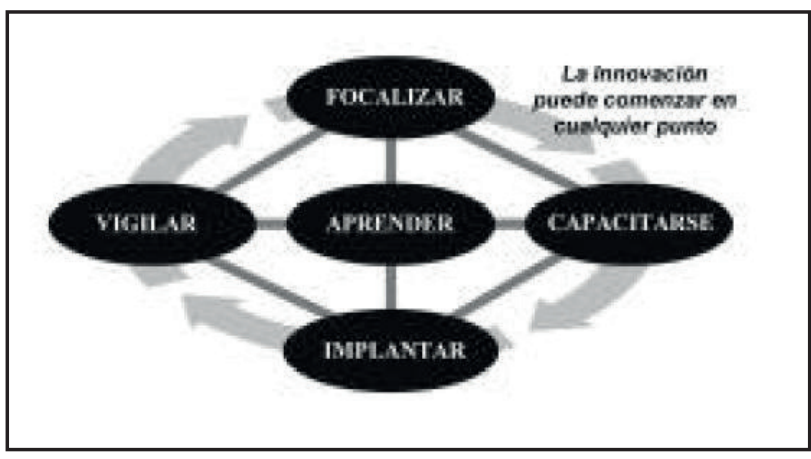

Gráfico 6. Modelo de gestión tecnológica

Temaguide.

Fuente: Fundación Cotec para la Innovación Tecnológica. 
estudio; el modelo Temaguide (ver gráfico 6) se acomoda a sus necesidades y se estima que este pueda replicarse a la mayoría de mypimes del país.

Para dicha implementación ha sido necesario el uso de algunas herramientas contempladas para el desarrollo del modelo.

\section{Vigilar}

- Análisis DOFA: permite realizar un análisis interno y externo. Su aplicación es esencial para conocer los desafíos a los que se enfrenta una organización.

- Análisis de la competencia: relacionado con investigación de documentación en cuanto a las patentes y bases de datos con el fin de establecer quién está haciendo las cosas y dónde las aplica.

- Benchmarking: selección de buenas prácticas del sector y compararse con ellas.

\section{Focalizar}

- Análisis de las cinco fuerzas de Porter: herramienta valiosa para determinar la estrategia competitiva de la empresa. Las cinco fuerzas son: la rivalidad entre empresas, el poder de negociación con clientes, el poder de negociación de proveedores, la amenaza de productos sustitutos y la amenaza de nuevos competidores.

- Auditoría de capacidades: identificar las capacidades de la compañía, es decir, qué es lo que puede hacer bien y qué no.

- Diagrama causa y efecto: método gráfico que permite identificar los factores que pueden derivar en futuros problemas para la empresa.

\section{Capacitar}

- Gestión de proyectos: cualquier implantación en la empresa debe tratarse y gestionarse como un proyecto. Debe tener objetivos claros, recursos debidamente asignados, con un tiempo de duración establecido y un director de proyecto encargado.

\section{Implantar}

Gestión del cambio: forma estructurada de gestión en la organización para adaptarse a los cambios.

Mejora continua: basada en perseguir cualquier oportunidad de mejora propuesta por los empleados.

\section{Aprender}

En la búsqueda de evaluación de las herramientas aplicadas en los elementos anteriores.

\section{Relación diagnóstico-modelo}

El modelo Temaguide es ideal para el funcionamiento de la empresa observada, así como la aplicación de sus herramientas específicas se alinea con su situación actual y su planeación estratégica direccionada a la competitividad y la mejora continua.

La organización analizada nunca contempló la necesidad de la tecnología como base para su gestión y menos la de la gestión de su tecnología; esto hace que el modelo Temaguide responda a la ausencia actual de Gestión Tecnológica y a los requerimientos futuros derivados de su aprendizaje.

\section{Indicadores de gestión}

La forma para complementar el modelo de Gestión Tecnológica es la elaboración de indicadores que permitan a la organización medirlo y controlarlo. Los indicadores propuestos son:

Seguimiento integral a los proveedores: (total proveedores con calificación satisfactoria/total de proveedores evaluados ${ }^{\star} 100$. Este indicador mide el impacto del sistema relacionándolo con el poder de negociación con los proveedores y las ventajas competitivas que se obtengan.

Cumplimiento de entregas al cliente: ( $\sum$ días de retraso/número de órdenes programadas). Permite 
medir el cumplimiento y estimar el poder de negociación con los clientes.

Cumplimiento del programa de auditorías de calidad: ( $\sum$ días de retraso/total auditorías programadas). Mide la disciplina de la organización con sus políticas de mejora continua.

Seguimiento general de indicadores (total de indicadores cumplidos/total de indicadores medidos): permite una misión global del modelo.

\section{Conclusiones}

La consulta de la literatura relacionada acompañada del diagnóstico a una mypime colombiana del sector de manufacturas permite de forma teórica validar el Modelo Temaguide como solución útil para la Gestión Tecnológica en la búsqueda de ventajas que permitan que esta tenga características diferenciadoras, vitales para ser competitivas frente a las diversas empresas con las que compite diariamente.

Se estima que en el lapso de un año los resultados sean los esperados, lo que significa que dicho modelo genere dentro de la empresa nuevos métodos y herramientas que evidencien mejoras en la empresa.

Un resultado positivo indica la posibilidad de implementar dicho modelo dentro de las mypimes colombianas. La importancia de la gestión tecnológica es evidente y el método Temaguide se vislumbra como el camino que permita vincular la planeación estratégica con la administración de la innovación.

\section{Referencias}

Agudelo, E. M., Niebles, L. M., Gallón, L. (2005). La gestión tecnológica como herramienta de planeación estratégica y operativa para las unidades de información. Revista Interamericana. Biblioteca Medellín (Colombia), 28(2), 89-114.

Fundación Cotec para la Innovación Tecnológica. (1999). Temaguide: pautas metodológicas en gestión de tecnología y de la innovación de empresas. Módulo I. Perspectiva empresarial. Madrid.

Test de Innovación Empresarial ICT. Instituto Catalán de Tecnología. (1999). Recuperado dewww.cea.es 